\title{
Germanica
}

\section{La mode intellectuelle du dilettantisme aux alentours de 1890 à Vienne et le jeune Hofmannsthal}

Der Dilettantismus in Wien um 1890 und der junge Hofmannsthal

Joëlle Stoupy

\section{(2) OpenEdition}

Journals

Édition électronique

URL : http://journals.openedition.org/germanica/556

DOI : 10.4000/germanica.556

ISSN : 2107-0784

Éditeur

Université de Lille

Édition imprimée

Date de publication : 1 décembre 2008

Pagination : 65-74

ISBN : 978-2-913857-22-6

ISSN : 0984-2632

\section{Référence électronique}

Joëlle Stoupy, "La mode intellectuelle du dilettantisme aux alentours de 1890 à Vienne et le jeune Hofmannsthal », Germanica [En ligne], 43 | 2008, mis en ligne le 01 décembre 2010, consulté le 06 octobre 2020. URL : http://journals.openedition.org/germanica/556 ; DOI : https://doi.org/10.4000/ germanica.556

Ce document a été généré automatiquement le 6 octobre 2020.

(c) Tous droits réservés 


\section{La mode intellectuelle du dilettantisme aux alentours de 1890 à Vienne et le jeune Hofmannsthal}

Der Dilettantismus in Wien um 1890 und der junge Hofmannsthal

Joëlle Stoupy

Le jeune Hermann Bahr arrive à Paris le 16 novembre 1888. Devant l'agitation qu'il y rencontre, il a l'impression, dit-il, de ressembler à un paysan qui aurait quitté son coin

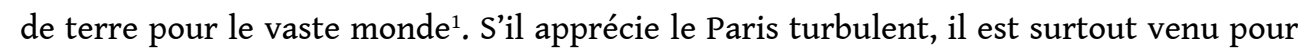
connaître la vie intellectuelle de la capitale française. À son retour à Vienne, Hermann Bahr contribuera à propager les dernières modes intellectuelles auxquelles il aura été confronté à Paris. Les essais qu'il écrira lors de son séjour à Paris et à la suite de ce séjour fourmillent de noms d'auteurs français alors en vogue comme Barrès, Maeterlinck, Huysmans, Villiers de l'Isle-Adam, Edouard Rod, Catulle Mendès ou Paul Bourget. Paul Bourget notamment est, à cette date en France, une célébrité. Il a derrière lui la publication des Essais et Nouveaux Essais de psychologie contemporaine (1883-1886). Le critique Antoine Albalat se rappelle dans ses Souvenirs de la vie littéraire le succès de ces essais. Il écrit : « La jeunesse française de 1883 à 1887 fut très fortement influencée par les retentissants débuts de M. Paul Bourget. Les Essais et Nouveaux Essais de psychologie contemporaine provoquèrent toute une nouvelle orientation dans l'esprit de ceux qui [...] se préparaient alors obscurément à la production littéraire $»^{2}$. Hermann Bahr s'intéresse, entre autres, à une attitude intellectuelle que Paul Bourget a définie le premier dans son essai sur Renan et qui fascine de nombreux auteurs français comme Anatole France, Edouard Rod, Maurice Barrès ou Jules Lemaitre : le dilettantisme. Pour beaucoup, Paul Bourget est alors celui «qui a mis à la mode le dilettantisme $»^{3}$, comme le dit le critique René Doumic en 1898. Bourget en parle sous de multiples formes dans ses essais, et il l'illustre aussi dans ses romans par des personnages aujourd'hui oubliés comme Claude Larcher, Robert Greslou ou Julien Dorsenne. La définition du dilettantisme de Bourget a, en son temps, marqué les esprits. Le dilettantisme, écrit Bourget, est une «disposition de l'esprit, très intelligente à la fois et très 
voluptueuse, qui nous incline tour à tour vers les formes diverses de la vie et nous conduit à nous prêter à toutes ces formes sans nous donner à aucune $»^{4}$. En donnant cette définition, Bourget ajoute que le concept de dilettantisme est chatoyant. Le dilettantisme est la "facilité à tout admettre des contradictions de l'univers ", le rêve du dilettante étant « d'avoir une âme à mille facettes pour réfléchir tous les visages de l'insaisissable Isis $»^{5}$. Le dilettantisme est aussi refus de toute limite. La règle de vie est l'épanouissement du moi, comme l'a montré Maurice Barrès dans son Culte du moi. L'obsession du dilettante est d'embrasser par la pensée tous les aspects de la réalité, réaliser plusieurs vies différentes. Henri-Frédéric Amiel, à qui Paul Bourget consacrera un essai dans lequel il montrera les méfaits du dilettantisme, écrit dans son journal: "Comprendre toutes les existences et toutes les formes de l'activité humaine m'a été plus cher que d'en réaliser une, arbitrairement choisie " ${ }^{6}$. Car le choix s'avère être un sacrifice, alors que «comprendre tout permet de goûter à tout $»^{7}$. Tel est le rêve de Julien Dorsenne dans Cosmopolis, le roman de Paul Bourget :

[...] il rêvait d'éprouver de l'existence humaine le plus grand nombre des impressions qu'elle peut donner et de les penser après les avoir éprouvées. [...]. Sa constante étude avait donc consisté [...] à traverser le plus de milieux différents qu'il lui avait été possible. Mais il les avait traversés en s'y prêtant sans jamais s'y donner, avec cette idée toujours présente dans l'arrière-fonds de son esprit qu'il existait de par ailleurs d'autres mœurs à connaître, d'autres caractères à regarder, d'autres personnages à revêtir, d'autres sensations sous lesquelles vibrers.

2 La vie dans les grandes villes et notamment à Paris semble à Bourget favorable au dilettantisme. Il écrit dans l'essai sur Renan à ce propos :

Cette ville est le microcosme de notre civilisation. [...] Dites maintenant s'il est possible de se conserver une unité de sentiments dans cette atmosphère surchargée d'électricités contraires, où les renseignements multiples et circonstanciés voltigent comme une population d'invisibles atomes. Respirer à Paris, c'est boire ces atomes, c'est devenir critique, c'est faire son éducation de dilettante ${ }^{9}$.

3 Si la critique de Bourget envers le dilettantisme reste modérée dans les Essais de psychologie contemporaine, s'il montre même envers cette attitude intellectuelle une certaine attirance, il connaît peu de temps plus tard une opposition sans concession à ce qu'il appelle en 1893 dans Cosmopolis «le fond du fond de la décadence ${ }^{10}$. Déjà en 1889, dans son roman Le Disciple, il condamne le dilettante qui « a fait le tour de toutes les idées » en lui reprochant de ne croire qu'au " jeu amusé de son esprit »"${ }^{11}$. Il voit en lui « un égoïste subtil et raffiné dont toute l'ambition [...] consiste à 'adorer son moi', à le parer de sensations nouvelles $»^{12}-$ un "jongleur d'idées » qui "abuse du monde intellectuel et sentimental $»^{13}$.

4 Si Paris semble prédestiné à encourager le dilettantisme, Hermann Bahr ne s'en plaint pas. Il ressent la métropole française comme un lieu, « où il suffisait d'ouvrir ses nerfs et de laisser couler en soi la ronde avide des impressions riches $»^{14}$. Très vite, il devient attentif aux nouveaux mots d'ordre dans la capitale française. Par opposition à la scène culturelle berlinoise qu'il connaît, de nouveaux concepts s'offrent à lui à Paris ; on parle de crise du naturalisme, de symbolisme, de psychologisme. Les études conçues à Paris reflètent l'intérêt que porte Hermann Bahr au dilettantisme - une attitude qui d'ailleurs semble lui avoir été familière dès l'adolescence, car il affirme avoir connu dans sa jeunesse une certaine "volubilité de penser ${ }^{15}$ qui le poussait à tout prouver en ne croyant à rien. Dans ses œuvres parisiennes, ses personnages pratiquent le dilettantisme. Il y a par exemple le personnage d'Heinrich Hönig dans son esquisse Die Märtyrer der Liebe, datant de 1889, un jeune Viennois vivant à Paris que Bahr appelle 
" un sceptique moderne $»^{16}$. Il connaît, comme un peu plus tard le personnage de son roman Die gute Schule, la manie de la contradiction, le doute systématique, l'introspection excessive. S'il envie le bonheur de ceux qui croient en quelque chose, il ne peut s'en satisfaire. Il ressemble au dilettante de Paul Bourget, Claude Larcher dans le roman Mensonges dont il est dit: «Comme beaucoup de sceptiques de nos jours, il soupirait sans cesse vers la simplicité de la foi [...] et sans cesse le goût des complexités intellectuelles ou sentimentales lui montrait dans une foi, quelle qu'elle fût, une mutilation, il n'osait ajouter: une bêtise $»^{17}$. Hermann Bahr connaît aussi ce que Bourget a appelé le "dilettantisme du vagabondage $»^{18}$ qui consiste en un cosmopolitisme raffiné et voluptueux, à la recherche de nouvelles sensations que procurent les voyages. Bahr en fait état dans deux études Das spanische Buch en 1889 dans laquelle il annonce vouloir faire du "sensationnisme ${ }^{19}$ et dans Russische Reise en 1891 dans laquelle le modèle de Maurice Barrès est prédominant. Bahr veut en Russie avoir une âme russe. "Je voulais emprunter à ce peuple sa relation au monde, pour m'assimiler ses plaisirs $»^{20}$, écrit-il dans Russische Reise. Pour le critique suédois Ola Hansson en 1891, Bahr est le type même de ce que l'on appelle en France un dilettante. Il sait entrer dans les cultures et les personnalités les plus contradictoires «avec la mine triomphante d'un acrobate, d'un homme caoutchouc qui tord ses membres dans les positions les plus incroyables $»^{21}$.

5 Fin mars 1890, Bahr quitte Paris en direction de Vienne. Mais une fois à Vienne, il reçoit la proposition d'Arno Holz de collaborer à Berlin à la revue Freie Bühne für modernes Leben. Le $1^{\mathrm{er}}$ mai 1890 , Bahr est à Berlin. Il y fait la connaissance des grands noms de la scène littéraire berlinoise comme Fontane, Spielhagen ou Liliencron. Il affirme cependant bientôt plein de dépit connaître certes toutes les célébrités de Berlin, mais cette ville lui parait maintenant, après ses expériences parisiennes, en retard de deux siècles $^{22}$. Berlin est pour lui toutefois une station importante dans sa production littéraire. Il trouve en Samuel Fischer un éditeur prêt à publier son roman des états d'âme Die gute Schule - ce qui lui fait dire qu'à présent, on ne pourra plus passer son nom sous silence. Parce qu'il est confronté cependant à des problèmes d'argent à Berlin, son père lui propose de retourner à Vienne. Mais Vienne lui apparaît pour l'instant peu attractive, il n'est là-bas personne, dit-il dans une lettre à son père et serait relégué à l'arrière-plan. Or, c'est un premier rôle qu'il recherche. Après son voyage en Russie, Bahr est toutefois de retour à Vienne en avril 1891 et il espère y jouer cette fois-ci un rôle déterminant. Il a fait, quelque temps auparavant, par lettre, la connaissance d'Eduard Michael Kafka, ce jeune homme de lettres viennois, désireux de donner un nouvel essor à la littérature autrichienne par sa revue Die Moderne Dichtung qui compte parmi les faits marquants de la constitution du groupe Jung Wien. Dans le texte Zehn Jahre, Bahr évoque la lettre «fougueuse et agressive " que lui fit parvenir Eduard Michael Kafka dans l'espoir d'une collaboration. Bahr devient bientôt «l'organisateur " de Jung Wien. Dans son pamphlet Die demolirte Literatur, Karl Kraus décrit le changement qui se produisit au café Griensteidl, bientôt sous le signe du symbolisme et de l'observation des états d'âme. Comme nous l'apprend l'autobiographie d'Hermann Bahr, c'est aussi la personnalité d'Hugo von Hofmannsthal et ses débuts prometteurs qui incitent Bahr à rester à Vienne et à y trouver une sphère d'influence. Son portrait du jeune Hofmannsthal, connu sous le titre Loris, nous décrit l'effet inoubliable que fit sur lui la lecture de l'essai d'Hofmannsthal, Die Mutter. Bahr est comblé en voyant que ce jeune artiste qu'il ne connaît pas encore personnellement a su se glisser dans sa personnalité avec une sensibilité et une souplesse étonnantes et 
la comprendre intimement, à la façon des critiques français, d'un Lemaitre ou d'un Bourget. Il y décrit le jeune Hofmannsthal comme un dilettante de grande envergure. Qu'il s'agisse d'un «simple Viennois", comme dit Bahr, l'étonne, tant il voit Hofmannsthal façonné par l'esprit français fin de siècle.

Avant qu'il ne fasse la connaissance d'Hermann Bahr le 27 avril 1891, Hofmannsthal connaissait cependant déjà ses écrits, car il est très tôt réceptif à son époque. Dans une lettre d'août 1890 à Gustav Schwarzkopf, il nomme sur un ton enjoué, les noms qu'il faut connaître pour ne pas être vieux jeu, pour être dans l'esprit fin de siècle - Wagner et Nietzsche, Böcklin et Brillat-Savarin, Paul Bourget et Schopenhauer ${ }^{23}$. Si Hofmannsthal connaissait le dilettantisme par la lecture de Bourget, Hermann Bahr a certainement contribué par sa personnalité à le lui rendre plus présent - Hofmannsthal écrit par exemple dans son journal le 25 mai 1891 s'être promené " presque trois heures $»^{24}$ avec Bahr au Volksgarten et l'avoir écouté parler entre autres de Bahr, Barrès et Bourget. Mais Hofmannsthal a trouvé aussi dans les œuvres de Nietzsche une réflexion sur l'abus de l'histoire qui, par certains aspects, rappelle la critique du dilettantisme que fait Bourget. Il retrouve d'ailleurs Nietzsche dans les aphorismes de Bourget, une ressemblance qui lui paraît à juste titre " involontaire $»^{25}$, car Bourget ne connaissait à cette date vraisemblablement pas Nietzsche. Une lecture des premières œuvres d'Hofmannsthal montre que l'attitude intellectuelle du dilettantisme le préoccupe lui aussi. Les premiers essais montrent par exemple le souci de débattre notamment de la "manie de la dissection intime " propre au dilettantisme. Le drame lyrique Gestern à propos duquel Hofmannsthal dira à Hermann Bahr que son personnage principal, Andrea, est un dilettante, tente de vérifier le mode d'existence que réclame le dilettantisme. En laissant son personnage échouer, Hofmannsthal inaugure ses doutes envers le dilettantisme. Claudio dans Der Tor und der Tod montre, lui, les conséquences du dilettantisme. À côté de l'esthète, Claudio représente le dilettante qui n'a jamais su se donner. Il a été spectateur de la vie, l'a « comprise », l'a vécue « comme un livre ${ }^{26}$. Les autres n'ont été pour lui que des "poupées », des « jouets », lui-même un « éternel joueur $\aleph^{27}$, un "mauvais comédien $»^{28}$ à qui la mort apprendra le respect de la vie. Souvent dans ses lettres ou ses notes des années 1890, Hofmannsthal parle lui-même de son manque d'immédiateté, de son habitude à se regarder vivre, de son intoxication littéraire. Il explique à son ami Edgar Karg von Bebenburg comment on se sent alors : " on est comme un fantôme en plein jour, on sent vivre en soi des pensées étrangères, des états d'âme anciens, morts, artificiels, on voit les choses comme derrière un voile, on est comme exclu de la vie, rien ne touche, rien ne comble vraiment ${ }^{29}$. Dès son premier essai consacré à la Physiologie de l'amour moderne de Bourget, Hofmannsthal a l'occasion de parler d'un dilettante. Il s'agit de Claude Larcher qu'Hofmannsthal connaissait déjà du roman Mensonges et que Bourget décrit comme suit :

Comme beaucoup d'écrivains d'analyse, il [Claude Larcher] était habitué à s'étudier et à se juger sans cesse, étude et jugement qui n'avaient d'ailleurs aucune influence sur ses actions. Les plus menus détails lui servaient de prétextes à des retours sur lui-même et sur sa destinée, mais le seul résultat de ce dédoublement continuel était de l'entretenir dans une lucidité inefficace et douloureuse de tous les instants ${ }^{30}$.

Hofmannsthal aurait pu, dans son essai sur Bourget, s'arrêter sur les aphorismes autour du thème de l'amour moderne et de ses caractéristiques qui font en fait l'objet des méditations de Bourget dans La Physiologie de l'amour moderne. Mais il est surtout attentif au personnage de Claude Larcher et à l'incapacité de ce dernier à trouver en lui 
l'unité - une incapacité qui résulte de la manie qu'il a de s'observer. Ainsi, comme Andrea dans Gestern, Claude Larcher connaît le divorce entre son moi d'hier et celui d'aujourd'hui. Comme Henri-Frédéric Amiel qui bientôt occupera Hofmannsthal, Claude Larcher raconte "l'histoire douloureuse d'un moi dédoublé $»^{31}$ et celle d'un artiste qui veut plus qu'il ne peut. Quant à Bourget, le jugement d'Hofmannsthal est dès 1891 irrémédiable. Même si Hofmannsthal puise de nombreuses idées dans ses textes, il n'est pas dupe: Paul Bourget n'échappe pas au snobisme. "Il flotte un parfum si aristocratique de cercle et de mirliton, de bookmakers et de marquises dans ce livre ${ }^{32}$, dit Hofmannsthal à propos de La Physiologie de l'amour moderne.

8 C'est sous les dehors d'un dilettante qu'Hofmannsthal appréhende aussi Hermann Bahr dans son essai Die Mutter. Il remarque en lui l'idée de multiplicité propre au dilettantisme. Hermann Bahr, dit Hofmannsthal d'un ton qui semble à la fois réprobateur et plein d'empathie, se laisse pousser par mille courants. Pour réfléchir tous les aspects de la vie, Bahr n'est plus qu'un « regard ouvert sur le vaste monde riche d'une multitude de formes $»^{33}$. Dans son volume d'essais Kritik der Moderne, Hermann Bahr pratique cependant pour Hofmannsthal déjà l'éparpillement: "Bahr à Vienne, Bahr à Berlin, Bahr à Paris, Bahr chez Ibsen, Bahr au Louvre $»^{34}$, Hofmannsthal voit ici le désir ou l'obsession de Bahr de décrire au plus près chaque atome de son existence. Son esprit qui aurait pu être "un grand miroir de la vie » ${ }^{35}$, Bahr l'a brisé en mille fragments. Hofmannsthal dit par ailleurs le mal qu'il a à croire aux personnages de Bahr et à leur souffrance et rejette ici de façon expressive le dilettantisme qu'il qualifie de «maladie de la sensibilité $»^{36}$. Une seule scène dans la pièce de théâtre de Bahr lui semble ressentie, la scène dans laquelle le personnage du fils, Edi, évoque sa nostalgie de la vie simple, son désir d'être comme ceux qui « ne savent rien [...] mais qui sont heureux $»^{37}$.

9 La même année, le dilettantisme apparaît à Hofmannsthal aussi sous les traits du professeur de philosophie suisse, Henri-Frédéric Amiel, un essai directement inspiré de celui de Bourget. L'épigraphe - «Oh, qu'un peu de bonheur naif est une douce chose !» - énonce déjà le problème qui tourmente Amiel. S'observant avec minutie et poussé par sa soif de l'infini qu'il a puisée dans la pensée allemande, Amiel a fini par perdre pied dans la réalité. Il s'avère incapable de supporter les limites de l'existence, lui qui rejette «l'enfant du hasard, la réalité $»^{38}$ pour goûter à tous les possibles. Hofmannsthal s'intéresse surtout à Amiel, car son âme de dilettante aurait presque pu faire de lui un artiste. Or, à ses yeux, il n'en est pas un. Certes, il possède de nombreuses qualités propres à l'artiste, il connaît les douleurs profondes, il sait sentir l'âme des choses, il a le don du mot juste, de la nuance. Mais Amiel n'a pas la force de vouloir, il est un « Raphael sans mains ». La conclusion d'Hofmannsthal est sévère et sans appel : « Cette surabondance est en fait un manque ; tout vouloir n'est rien d'autre que l'incapacité de se limiter $»^{39}$. Contrairement à l'artiste, Amiel ne sait pas fermer les yeux devant la multitude d'alternatives qui s'offrent à lui et créer. Leopold von Andrian se souvient plus tard avoir souvent parlé dans ces années-là avec Hofmannsthal de l'antagonisme entre artiste et dilettante. Le dilettante leur apparaissait à tous deux comme un "personnage ridicule», dit Andrian. Hofmannsthal parlait volontiers de tous ceux qui ne savaient pas réaliser une œuvre et en restaient au stade des bonnes intentions ${ }^{40}$.

10 Dès 1891 , le dilettantisme semble à Hofmannsthal être, somme toute, un «jeu dangereux avec son élasticité $»^{41}$. Se limiter est un $"$ devoir $»^{42}$ écrit-il en 1891 dans ses notes, comme pour se prémunir contre un danger latent. En terminant la lecture des 
journaux d'Amiel, il note : "On quitte ce livre avec une nostalgie ineffable pour ce qui est limité, pour les contours précis, pour la netteté tangible et commune $»^{43}$. L'essai Die Menschen in Ibsens Dramen datant de 1892 nous montre qu'Hofmannsthal mesure à présent régulièrement l'art contemporain à l'aune du dilettantisme. Dans les drames d'Ibsen, il rencontre des dilettantes qui ont le pouvoir et la manie de l'introspection et une des questions qu'il se pose est de savoir si la manie de l'analyse et la vie font bon ménage. Hofmannsthal doit reconnaître que les personnages d'Ibsen vivent, comme Amiel, une vie fantomatique. Ils ne connaissent presque pas l'action et vivent de pensées et d'états d'âme. Cette analyse excessive de la vie, Hofmannsthal en fait la marque distinctive de son époque dans son premier essai sur Gabriele d'Annunzio. Il dira dans cet essai sur d'Annunzio en 1893 que cette attitude est commune à sa génération ou, plus exactement, à «quelques milliers de personnes rassemblées dans les grandes villes européennes $»^{44}$. Dans cet essai, il déplore que sa génération n'ait pas de «racines dans la vie $»^{45}$. En 1894, il écrit alors à Edgar Karg von Bebenburg son propre désir de passer d'une attitude dilettante face à l'art à un arrangement minutieux avec la vie. Avec plus de sérieux que Bahr, Hofmannsthal s'est confronté au dilettantisme dont il dira plus tard qu'il contient « le germe d'une corruption morale » ${ }^{46}$.

\section{NOTES}

1. «[...] noch bin ich betäubt, wie in einem Taumel, ganz außer mir, einem Bauer zu vergleichen, der aus seinem einsamen Dorf in die große Welt tritt » (H. Bahr: Briefwechsel mit seinem Vater. Ausgewählt von Adalbert Schmidt, Wien, H. Bauer-Verlag, 1971, p. 200.

2. A. Albalat: Souvenirs de la vie littéraire, Paris, Crès, 1924, p. 207.

3. R. Doumic: Ecrivains d'aujourd'hui, Paris, Perrin, 1898, p. 10.

4. P. Bourget: Essais de psychologie contemporaine, Paris, Lemerre, 1883, p. 59.

5. Ibid., p. 63.

6. H. F. Amiel: Journal intime, tome II, Paris, Stock, 1927, p. 72-73.

7. J.-F. Hugot: Le Dilettantisme dans la littérature française d'Ernest Renan à Ernest Psichari, Paris, Aux Amateurs de livres, 1984, p. 284.

8. P. Bourget: Cosmopolis, Paris, Lemerre, 1894, p. 46.

9. P. Bourget: Essais de psychologie contemporaine, op. cit., p. 73-74.

10. P. Bourget: Cosmopolis, op. cit., p. 33.

11. P. Bourget: Le Disciple, Paris, Plon, 1901, p. 12.

12. Ibid., p. 12-13.

13. Ibid., p. 13.

14. " wo man bloß die Nerven aufzumachen und den gierigen Reigen reicher Impressionen in sich hineinrieseln zu lassen brauchte» in : H. Bahr: Prophet der Moderne. Tagebücher 1888-1904. Ausgewählt und kommentiert von Reinhard Farkas, Wien, Graz, Köln, Böhlau, 1987, p. 30.

15. «Volubilität des Denkens »; « Ich konnte alles beweisen und glaubte eigentlich gar nichts [....] Dies wurde mir so geläufig, daß ich spielend alles aufnehmen konnte, ohne davon im Innern behelligt oder auch nur berührt zu werden » in : H. Bahr: Das Hermann-Bahr-Buch zum 19. Juli 1913, Berlin, S. Fischer, 1913, p. 91. 
16. Cf. Joëlle Stoupy: 'Maître de l'heure'. Die Rezeption Paul Bourgets in der deutschsprachigen Literatur um 1890, Frankfurt/Main, Lang, 1996, p. 84.

17. P. Bourget: Mensonges, Paris, Lemerre, 1890, p. 513.

18. P. Bourget: Essais de psychologie contemporaine, op. cit., p. 305.

19. H. Bahr: Prophet der Moderne, op. cit., p. 53.

20. «Ich wollte diesem Volke sein Verhältnis zur Welt entnehmen, um mir seine Genüsse anzueignen » in : H. Bahr: Russische Reise, Dresden und Leipzig, E. Pierson's Verlag, 1891, p. 84.

21. «Er ist der Typus dessen, was der Franzose Dilettantismus nennt [...]. Er thut das nicht bloß bewußt, wie z.B. Bourget und im allgemeinen alle modernen Kritiker, die von Taine ausgegangen sind, sondern er thut es mit der ganzen triumphierenden Miene eines Akrobaten, eines Kautschukmenschen, der seine Glieder in die unmöglichen Stellungen verdreht hat " in : 0 . Hansson: «Neue Bücher», in: Freie Bühne für modernes Leben, II. Jg. 1891, p. 1125.

22. «Ich kenne bald alle 'Berühmtheiten' von Berlin, aber im Vergleich mit meinem Pariser Packträger sind sie nur Trotteln, ohne Ausnahme. Dieses ganze Volk, oben und unten, ist um zwei Jahrhunderte zurück» in : H. Bahr: Prophet der Moderne, op. cit., p. 33-34.

23. H. von Hofmannsthal: Lettre à Gustav Schwarzkopf, cf. J. Stoupy: 'Maître de l'heure', op. cit. , p. 121.

24. H. von Hofmannsthal: Reden und Aufsätze III. 1925-1929. Aufzeichnungen, Frankfurt/Main, Fischer Taschenbuch Verlag, 1980, p. 330.

25. H. von Hofmannsthal: Reden und Aufsätze I. 1891-1913, Frankfurt/Main, Fischer Taschenbuch Verlag, 1979, p. 96.

26. «Mein Leben zu erleben wie ein Buch » in : H. von Hofmannsthal: Gedichte. Dramen I. 1891-1898, Frankfurt/Main, Fischer Taschenbuch Verlag, 1979, p. 284.

27. Ibid., p. 294.

28. Ibid., p. 296.

29. «[...] man ist wie ein Gespenst bei hellem Tage, fremde Gedanken denken in einem, alte künstliche Stimmungen leben in einem, man sieht die Dinge wie in einem Schleier, wie fremd und ausgeschlossen geht man im Leben herum, nichts packt, nichts erfüllt einen ganz» in : H. von Hofmannsthal: Briefe 1890-1901, Berlin, S. Fischer, 1935, p. 77.

30. P. Bourget: Mensonges, op. cit., p. 4-5.

31. «Leidensgeschichte eines gespaltenen Ich» in : H. von Hofmannsthal: Reden und Aufsätze I, op. cit., p. 115.

32. «Es weht ein so aristokratisches Parfum nach cercle und mirliton, bookmakers und Marquisen in dem Buch», ibid., p. 97.

33. « ein Blick in die ganze weite Welt voll reicher Formen », ibid., p. 100-101.

34. «Bahr in Wien, Bahr in Berlin, Bahr in Paris, Bahr bei Ibsen, Bahr im Louvre », ibid., p. 100.

35. « ein großer Lebensspiegel », ibid., p. 101.

36. « Krankheit des Empfindungsvermögens », ibid., p. 102.

37. «Weißt du, von ganz dummen Leuten - die sind glücklich; weit draußen, ganz am Ende... ja, sie wissen nichts und sind schlecht frisiert und haben keinen Geschmack, sondern häßliche, rote Hände, und wenn sie am Sonntag spazierengehen, dann lachen wir sie aus - aber die sind glücklich! », ibid., p. 105.

38. « das Zufallskind Wirklichkeit », ibid., p. 112.

39. «Dieser Überreichtum ist eigentlich Mangel; dieses Alleswollen nichts als die hilflose Unfähigkeit, sich zu beschränken », ibid., p. 114.

40. « der Dichter... Sein Gegensatz, der Dilettant dünkte uns eine lächerliche Figur. Hofmannsthal sprach gerne vom Dilettantengarten, und alle die Vielen, die ihre großartigen Absichten und Entwürfe nicht in die Tat des Kunstwerkes umzusetzen vermochten, ergingen sich darin » in : H. Fiechtner: Hugo von Hofmannsthal. Die Gestalt des Dichters im Spiegel der Freunde, Wien, Humboldt Verlag, 1949, p. 57. 
41. «dasselbe gefährliche Spiel mit seiner Elastizität» in : H. von Hofmannsthal: Reden und Aufsätze I, op. cit., p. 102.

42. H. von Hofmannsthal: Reden und Aufsätze III, op. cit., p. 321.

43. « 8.VI. - Amiels Tagebücher beendet: Man verläßt das Buch mit unsäglicher Sehnsucht nach Begrenztem, nach scharfen Konturen, nach greifbarer, gemeiner Deutlichkeit », ibid., p. 332.

44. «Wir! Wir! Ich weiß ganz gut, daß ich nicht von der ganzen großen Generation rede. Ich rede von ein paar tausend Menschen, in den großen europäischen Städten verstreut » in : H. von Hofmannsthal: Reden und Aufsätze I, op. cit., p. 175.

45. «Wir haben gleichsam keine Wurzeln im Leben und streichen, hellsichtige und doch tagblinde Schatten, zwischen den Kindern des Lebens umher », ibid.

46. «Im Dilettantismus ist der Keim einer sittlichen Verderbnis » in : H. von Hofmannsthal: Reden und Aufsätze III, op. cit., p. 285.

\section{RÉSUMÉS}

« Paris. Paris. Il faudrait que je l'écrive des milliards de fois pour exprimer ce que je ressens » écrit Hermann Bahr plein d'enthousiasme à propos de son premier voyage à Paris aux alentours de 1890. Le futur mentor de Jung Wien contribue à propager à son retour à Vienne les dernières modes intellectuelles auxquelles il a été confronté dans la capitale française et s'intéresse notamment à ce que l'on appellera le dilettantisme de la fin de siècle, une attitude intellectuelle que Paul Bourget a définie le premier dans son chapitre sur Renan issu des Essais de psychologie contemporaine (1883). Le jeune Hofmannsthal, réceptif à son époque, perçoit à son tour la modernité à travers le prisme du dilettantisme, une mode intellectuelle qu'il reconnaît chez de nombreux contemporains tel que Bahr, Ibsen ou Amiel. Dès 1891, elle lui apparaît cependant comme un «jeu dangereux » et on observe dans ses œuvres une distanciation par rapport à ce phénomène importé de Paris.

«Paris. Paris. Milliardenmale müßte ich es schreiben, um mein Gefühl auszudrücken» schreibt Hermann Bahr voller Begeisterung über seine erste Pariser Reise um 1890. Der spätere Mentor des Jungen Wien trägt bei seiner Rückkehr nach Wien dazu bei, die neuesten geistigen Moden zu propagieren, mit denen er in der französischen Hauptstadt in Berührung kam. Er interessiert sich u.a. für eine intellektuelle Erscheinung, den später genannten Dilettantismus des Fin de siècle, den Paul Bourget in seinem Kapitel über Renan in den Essais de psychologie contemporaine als erster definiert hat. Der junge Hofmannsthal befaßt sich ebenfalls mit dieser geistigen Erscheinung und erkennt den Dilettantismus an zahlreichen Persönlichkeiten wie Bahr, Ibsen, Barrès oder Amiel, aber auch an sich selbst. Schon 1891 erscheint ihm der Dilettantismus als «gefährliches Spiel mit seiner Elastizität» und man bemerkt eine Distanzierung von diesem Phänomen.

\section{INDEX}

Mots-clés : Vienne, dilettantisme 
AUTEURS

JOËLLE STOUPY

Université du Littoral-Côte d'Opale (Boulogne-sur-Mer) 